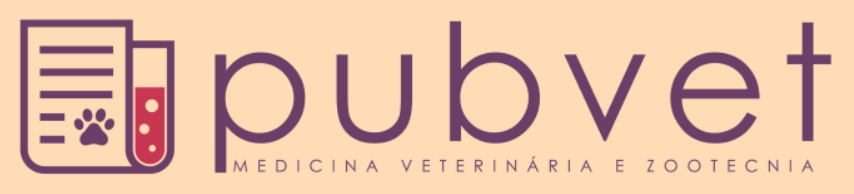

https://doi.org/10.22256/pubvet.v12n5a93.1-6

\title{
Qualidade físico-química e microbiológica de pescado congelado consumido na merenda escolar do estado do Amazonas
}

\author{
Paula Ribeiro dos $\operatorname{Santos}^{1 \bullet}$, Euclides Luis Queiroz de $\operatorname{Vasconcelos}^{1^{*} \odot}$, Antônio Fábio \\ Lopes de Souza ${ }^{2} \bullet$, Joel Lima da Silva Júnior ${ }^{3}$, Antônio José Inhamuns ${ }^{4} \bullet$ \\ ${ }^{I}$ Acadêmicos de Engenharia de Pesca, Faculdade de Ciências Agrárias (FCA), Universidade Federal do Amazonas (UFAM) \\ ${ }^{2}$ Doutor em Ciências Pesqueiras nos Trópicos -UFAM \\ ${ }^{3}$ Médico veterinário, Professor Doutor da FCA-UFAM \\ ${ }^{4}$ Engenheiro de Pesca, Professor Doutor da FCA-UFAM \\ *Autor para correspondência - e-mail: euclides_luis15@hotmail.com
}

RESUMO. O presente trabalho avaliou a composição físico-química e microbiológica de filés congelados de peixes regionais utilizados no cardápio das escolas da rede pública estadual de ensino. Foram analisadas 10 amostras de filés congelados referente às espécies Dourada (Brachyplatystoma rousseauxii), Mapará (Hypohythalmus spp.), Pirarara (Phractocephalus hemioliopterus), Piranambú (Pinirampus pirinampus) e Tucunaré (Cichla. spp.) obtidas no depósito da gerência de distribuição de merenda escolar da secretaria estadual de educação do Amazonas. Foram realizadas análises de umidade, proteínas, lipídios, cinzas e valor calórico, além de análises microbiológicas: coliformes totais e termotolerantes, Staphylococcus coagulase positiva, e presença/ausência de Escherichia coli e Salmonella spp. em $25 \mathrm{~g}$ de alimento. Os resultados microbiológicos encontrados foram comparados com os padrões microbiológicos para pescado e produtos de pescado que constam na legislação brasileira. Os filés avaliados evidenciaram bons aspectos nutricionais e microbiológicos, encontrando-se dentro dos parâmetros exigidos pela Agência Nacional de Vigilância Sanitária (ANVISA), estando aptos para o consumo da comunidade estudantil.

Palavras chave: Qualidade de alimentos, peixes regionais, alimentação escolar

\section{Physico-chemical and microbiological quality of frozen fish consumed in school lunch in the state of Amazonas}

\begin{abstract}
The present work evaluated the physical-chemical and microbiological composition of frozen fish fillets of regional fish used in the menu of the schools of the state public school system. Ten samples of frozen fillets Brachyplatystoma rousseauxii, Hypohythalmus spp., Phractocephalus hemioliopterus, Pinirampus pirinampus and Cichla spp. Were collected from the Distribution Management warehouse of School Lunch from the State Department of Education of Amazonas. Moisture, protein, lipids, ashes and calorific values were analyzed, as well as microbiological analyzes: total and thermotolerant coliforms, coagulase positive Staphylococcus, and presence / absence of Escherichia coli and Salmonella spp. in $25 \mathrm{~g}$ of food. The microbiological results were compared with the microbiological standards for fish and fish products that are included in the Brazilian legislation. The evaluated fillets showed good nutritional and microbiological aspects, being within the parameters demanded by the National Agency of Sanitary Surveillance (ANVISA), being classified as suitable for the consumption of the student community.
\end{abstract}

Keywords: Quality of food, regional fish, school feeding 


\title{
Calidad físico-química y microbiológica de pescado congelado consumido en la merienda escolar del estado de Amazonas
}

\begin{abstract}
RESUMEN. Con el presente trabajo se evaluó la composición físico-química y microbiológica de filetes congelados de peces regionales utilizados en el menú de las escuelas de la red pública estatal de educación. Se analizaron 10 muestras de filetes congelados de las especies Brachyplatystoma rousseauxii, Pirpohythalmus spp., Phractocephalus hemioliopterus, Pinirampus pirinampus y Cichla spp. Obtenidas del almacén de la gerencia de distribución de merienda escolar de la secretaría estadual de educación del Amazonas. Se realizaron análisis de humedad, proteínas, lípidos, cenizas y valor calórico, además de análisis microbiológicos: coliformes totales y termotolerantes, Staphylococcus coagulasa positiva, y presencia / ausencia de Escherichia coli y Salmonella spp. en $25 \mathrm{~g}$ de alimento. Los resultados microbiológicos encontrados fueron comparados con los patrones microbiológicos para pescado y productos de pescado que constan en la legislación brasileña. Los filos evaluados evidenciaron buenos aspectos nutricionales y microbiológicos, encontrándose dentro de los parámetros exigidos por la Agencia Nacional de Vigilancia Sanitaria (ANVISA), estando aptos para el consumo de la comunidad estudiantil.
\end{abstract}

Palabras clave: Calidad de alimentos, peces regionales, alimentación escolar

\section{Introdução}

A inclusão do pescado na merenda escolar pode incentivar o consumo deste pelas crianças, principalmente se incluído em preparações que os alunos apreciem. Este hábito pode representar uma maneira para aumentar o consumo do pescado no Brasil, e consequentemente a sua produção, visto que a pesca e a aquicultura, de maneira direta ou indireta, figuram papel fundamental nos meios de subsistência de milhões de pessoas em todo o mundo (Veit et al., 2012).

Apesar de todos os benefícios que o pescado apresenta, nutritivo, rico em micronutrientes, minerais, ácidos graxos essenciais do tipo ômega3 e ômega-6, de fácil digestibilidade, baixo teor de gordura, representa um valioso complemento nas dietas pobres em vitaminas e minerais essenciais (Fao, 2004; Silva et al., 2008).

É um alimento altamente suscetível a deterioração, devido a sua composição química e, sobretudo, o $\mathrm{pH}$ próximo à neutralidade, o que acaba favorecendo o desenvolvimento microbiano (Landgraf, 1996). Rosa et al. (2008) avaliaram as práticas de higiene durante a produção de preparações à base de carne em escolas públicas municipais na cidade de Natal/RN e, constataram que as condições higiênico-sanitárias das preparações à base de carne servidas na alimentação escolar apresentaram-se insatisfatórias.
A verificação da qualidade, tanto nutricional, como microbiológica constitui-se, numa importante etapa no processo de aquisição destes produtos para o consumo da comunidade estudantil, bem como pode contribuir de forma eficaz com a segurança alimentar dos estudantes da rede pública de ensino do estado do Amazonas.

\section{Material e métodos}

\section{Material}

Foram coletadas dez amostras de filés de pescado congelado, compreendendo as seguintes espécies: Dourada (Brachyplatystoma rousseauxii) Mapará (Hypophythalmus spp); pirarara ( Phractocephalus hemioliopterus); Piranambú ( Pinirampus pirinampus) e tucunaré (Cichla spp.)

Cada amostra pesou 1000 gramas.

\section{Metodologia da composição centesimal}

Foram realizadas em triplicata, retirando 100 gramas da amostra seguindo as normas preconizadas pela AOAC (1995) e adotadas pelo IAL (2008), que consta das seguintes determinações percentuais: umidade; cinza; lipídios; proteínas e valor calórico total. Os dados foram tratados estatisticamente pela análise de variância (ANOVA) em nível de $5 \%$ de significância. 


\section{Análise microbiológica}

As amostras de peixe congelado foram previamente descongeladas no refrigerador a uma temperatura de aproximadamente $4{ }^{\circ} \mathrm{C}$ por no máximo 18 horas. A metodologia analítica utilizada foi a recomendada pela Instrução Normativa $n^{\circ} 62$ de 26/08/2003 (Brasil, 2003) e Vasconcelos et al. (2014). Os resultados foram comparados com as normas preconizadas pela Agência Nacional de Vigilância Sanitária ANVISA (Brasil, 2003).

No laboratório foram retiradas 2 amostras analíticas (25 g cada), para cada análise, sendo separadamente tratadas: a partir de uma amostra analítica adicionada em $225 \mathrm{ml}$ de água peptonada $0,1 \%$, foram efetuadas três diluições sucessivas $\left(10^{-1}, 10^{-2}\right.$ e $\left.10^{-3}\right)$ para a quantificação de estafilococos coagulase positiva, coliformes termotolerantes e teste confirmativo de Escherichia coli.

Para determinação da presença/ausência de Salmonella spp., outra amostra analítica foi inoculada em $225 \mathrm{ml}$ de meio seletivo e transferida para outros meios seletivos diferenciais, incubados em diferentes temperaturas, de acordo com as etapas apresentadas por Brasil (2003) e Vasconcelos et al. (2014).

\section{Resultados e discussão}

As análises da composição centesimal (umidade, proteínas, lipídios totais, cinza, NIFEXT e energia Bruta) dos filés congelados servidos na merenda escolar no Estado do amazonas estão apresentados na tabela 1.

Tabela 1. Composição centesimal dos filés de peixe congelado.

\begin{tabular}{lccccc}
\hline \multicolumn{5}{c}{ Peixes } \\
\hline Fração & Dourada & Mapará & Pirarara & Piranambú & Tucunaré \\
\hline Umidade & $75,24 \pm 0,65^{\mathrm{a}}$ & $63,40 \pm 0,50^{\mathrm{d}}$ & $78,83 \pm 0,37^{\mathrm{b}}$ & $76,14 \pm 0,85^{\mathrm{c}}$ & $78,91 \pm 0,33^{\mathrm{b}}$ \\
Proteína & $19,7 \pm 0,37^{\mathrm{a}}$ & $14,95 \pm 0,86^{\mathrm{e}}$ & $18,67 \pm 0,43^{\mathrm{b}}$ & $17,23 \pm 0,08^{\mathrm{c}}$ & $16,25 \pm 0,29^{\mathrm{d}}$ \\
Lipídios & $3,03 \pm 0,23^{\mathrm{a}}$ & $20,13 \pm 0,15^{\mathrm{e}}$ & $0,73 \pm 0,048^{\mathrm{b}}$ & $4,65 \pm 0,23^{\mathrm{c}}$ & $1,61 \pm 0,31^{\mathrm{d}}$ \\
Cinza & $1,02 \pm 0,18^{\mathrm{a}}$ & $0,56 \pm 0,06^{\mathrm{b}}$ & $0,72 \pm 0,12^{\mathrm{a}}$ & $0,83 \pm 0,009^{\mathrm{a}}$ & $0,76 \pm 0,01^{\mathrm{a}}$ \\
NIFEXT* & $1,01^{\mathrm{a}}$ & $0,8^{\mathrm{a}}$ & $1,05^{\mathrm{a}}$ & $1,16^{\mathrm{b}}$ & $2,47^{\mathrm{c}}$ \\
Energia (Kcal EB/100g) & $110,11^{\mathrm{a}}$ & $244,17^{\mathrm{e}}$ & $85,45^{\mathrm{b}}$ & $115,41^{\mathrm{c}}$ & $89,37^{\mathrm{d}}$ \\
\hline
\end{tabular}

Fração não nitrogenada*

Média \pm desvio padrão; valores na mesma linha seguidos de letras diferentes, diferem entre si $(\mathrm{p}<0,05)$.

Na tabela acima observa-se que a umidade variou entre as espécies, sendo o menor valor encontrado, no filé de mapará $63,40 \%$ e maior percentual atribuído ao tucunaré, $78,91 \%$. Quando comparados com a Tabela Brasileira de Composição de Alimentos NEPA (2011), que relata percentuais médios de umidade de $78,6 \%$ nos filés de três espécies amazônicas dentre essas o filé de tucunaré, os resultados ficaram em consonância com os da literatura. Costa et al. (2010), ao estudarem o potencial do mapará como uma espécie alternativa para piscicultura na Amazônia determinaram percentuais de umidade para o filé de $65,18 \%$ e ventrecha de $63,8 \%$, superior ao encontrado no presente trabalho.

Os percentuais de proteínas observados foram elevados, mesmo com o filé de mapará apresentando menor percentual $(14,95 \pm 0,86)$. Veit et al. (2012) relatam que o pescado é excelente fonte de proteína animal, indispensável para crianças em fase de crescimento. Costa et al. (2010) observaram o valor $12,85 \%$ de proteína no filé de mapará, assim como Souza et al. (2013) estudando carne mecanicamente separada (CMS) de peixes regionais, dentre esses o mapará, e encontraram valores de $12,2 \%$, inferior ao obtido no presente estudo, os autores ressaltaram o alto teor de aminoácidos essenciais que estão presentes nas espécies regionais. NEPA (2011) mostra o valor de $18,8 \%$ de proteína para a espécie dourada (Brachyplatystoma rousseauxii) inferior ao encontrado no estudo para a mesma espécie.

Os lipídios totais apresentaram maior variação, com o menor percentual encontrado no filé de pirarara e o maior valor atribuído para o filé de mapará. Stansby \& Olcott (1968) elaboraram uma classificação para os peixes, uma das mais utilizadas até dos dias atuais, em que conforme a composição dos peixes, são agrupados em cinco classes, estando a dourada, pirarara, piranambú e tucunaré na categoria $\mathrm{A}$ (peixes com baixo teor de lipídios, menor que $5 \%$, e alto teor de proteínas 15 a $20 \%$ ), com 
exceção do filé de mapará, peixe altamente gordo de acordo com a classificação de Ackman (1989). Santiago et al. (2016) trabalhando com mapará encontrou $4,27 \%$ de lipídeos, no presente trabalho foi encontrado 20,13\%, valor bem superior. Entretanto Costa et al. (2010) determinaram $21,21 \%$ de lipídios para a espécie.

Estudos afirmam que os valores de lipídios em produtos cárneos podem passar diversas variações, que podem ser atribuídas a fatores como: idade, tipo de tecido, órgãos, sexo, maturidade sexual, regime alimentar ou estação sazonal (Yeannes \& Almandos, 2003). Esta relação entre o teor de gordura, proteína e água, encontrados para o mapará, diferindo de outras categorias de pescado da bacia amazônica, é justificável, uma vez que esta categoria não forma um depósito específico de gordura, sendo difundida em todo tecido muscular. Nesta categoria, assim como em todos os Siluriformes, a bexiga natatória é bastante reduzida, sendo a gordura essencial para sua flutuabilidade (Carvalho, 1980).

Os teores de carboidratos encontrados, estão de acordo com os dados em literatura para esse macronutriente. No presente estudo, o carboidrato variou entre 0,8 a $2,47 \%$. Souza et al. (2013) encontraram percentuais que variaram entre 0,56 e $0,94 \%$ inferiores ao encontrado no presente estudo.

Contreras-Guzmán (1994) citou que a fração de cinzas em peixes de água doce apresenta teores entre 0,90 e $3,39 \%$. Este valor é concordante com o teor de cinzas encontrado no presente trabalho para todos os filés das espécies analisadas. Ressaltando que a carne do pescado é considerada uma fonte valiosa de cálcio e fósforo, particularmente, apresenta também quantidades razoáveis de sódio, potássio, manganês, cobre, cobalto, zinco, ferro e iodo.

Em relação a energia, foi possível observar que o filé com alto teor energético apresentou maior conteúdo de gordura e baixa quantidade de água. Souza et al. (2013) determinaram para o mapará 186,67 Kcal de energia bruta/100g, essa diferença é justificável uma vez que os percentuais das frações centesimais determinada em seu trabalho foram menores.

$\mathrm{Na}$ tabela 2 estão apresentados os resultados das análises microbiológicas realizadas nos filés congelados de pescado regional servidos na merenda escolar da rede pública de ensino do Estado.

Na contagem de coliformes a $35^{\circ} \mathrm{C}$, utilizando o método de Número Mais Provável (NMP) verificou-se uma variação de $<10$ à $9,3 \times 10 \mathrm{NMP} /$ $\mathrm{g}^{-1}$. Quanto ao NMP de coliformes termotolerantes a $45^{\circ} \mathrm{C}$, foi detectada contaminação em três amostras (Dourada, mapará e pirarara).

Rall et al. (2008) estudaram a enumeração de coliformes termotolerantes em pescado congelado e fresco, e verificaram a presença em concentrações que variaram de $2,4 \times 10^{3}$ $\mathrm{NMP} / \mathrm{g}$ para o pescado congelado, concluindo que as amostras encontravam-se fora dos padrões recomendados pela Agência Nacional de Vigilância Sanitária, discordando dos resultados encontrado neste trabalho, que mantiveram-se em conformidade com a legislação vigente.

Tabela 2. Análise microbiológica dos filés de peixe congelado.

\begin{tabular}{|c|c|c|c|c|}
\hline \multirow[b]{3}{*}{ Peixes/Filés } & \multicolumn{4}{|c|}{ Microrganismos } \\
\hline & \multicolumn{2}{|c|}{$\left(\mathrm{NMP} . \mathrm{g}^{-1}\right)$} & \multirow{2}{*}{$\frac{\left(\mathrm{UFC} \mathrm{g}^{-1}\right)}{\mathrm{ECP}}$} & \multirow{2}{*}{$\begin{array}{c}\text { (PRES/AUS) } \\
\text { SALM }\end{array}$} \\
\hline & CT & $\mathrm{CF}$ & & \\
\hline Dourada & $4,3 \times 10$ & $<10$ & & \\
\hline Mapará & $<10$ & $2,3 \times 10$ & & \\
\hline Pirarara & $9,3 \times 10$ & $9,3 \times 10$ & AUS & AUS \\
\hline Piranambú & $2,3 \times 10$ & $<3$ & & \\
\hline Tucunaré & $2,3 \times 10$ & $<3$ & & \\
\hline Legislação (Brasil, 2001) & $10^{-2}$ (máx) & $10^{-2}$ (máx) & $10^{-3}$ (máx) & AUS \\
\hline
\end{tabular}


Os mesmos autores enfatizaram em seu trabalho que dentre as amostras de peixes, fresco ou congelado, coletadas como filé ou posta, observou-se que a contaminação por bactérias termotolerantes eram maiores em filés, pois o preparo deste inclui evisceração, retirada da pele e "filetagem", ou seja, exige maior manipulação quando comparado ao peixe fatiado em postas.

Assim como relataram Mukundan et al. (1986) e Vieira et al. (2004). No entanto, os valores encontrados no presente trabalho se mostraram superiores aos relatados por Dams et al. (1996), que ao avaliar a qualidade microbiológica da pescadinha (Cysnoscion striatus), inteira e em filés nos principais pontos críticos de controle de uma indústria de pescado congelado, não obteve a confirmação de coliformes termotolerantes, encontrando apenas coliformes totais. Contudo, mesmo encontrandose dentro dos padrões vigentes, foi possível observar que o filé de pirarara apresentou contagem bem superior em relação aos filés das demais espécies, tanto para coliformes totais quanto para termotolerantes.

Essa contaminação encontrada nas amostras, indicam uma provável falha durante o processamento, empacotamento e estocagem dos produtos. Landgraf (1996), relata que a pesquisa de coliformes termotolerantes nos alimentos fornece com maior segurança informações sobre condições higiênicas do produto e é empregado como indicador de contaminação pós-sanitização.

Em relação a contagem de estafilococos coagulase positiva, não foi observada contaminação em nenhuma das amostras. Farias \& Freitas (2008) avaliaram o pescado beneficiado em indústrias paraenses, verificaram que, em $50(98,0 \%)$ e $1(2,0 \%)$ amostras de peixes eviscerados congelados e 53 $(98,1 \%)$ amostras e uma $(1,9 \%)$ amostras de filés de peixes congelados atenderam e não atenderam, respectivamente, ao padrão estabelecido pela legislação federal, com máximo de 1030 UFC/g. Assim sendo, a negatividade do referente microrganismo no presente estudo indica que o nível de higiene na manipulação durante o processamento foi satisfatório.

Não foi evidenciada a presença de Salmonella spp. nas amostras analisadas, atendendo ao padrão de ausência em $25 \mathrm{~g}$ estabelecido pela legislação (Brasil, 2001).
Segundo Ahmed (1991), o pescado é um veículo de salmonelose muito menos frequente do que outros produtos alimentares, sendo o peixe e os mariscos responsáveis apenas por uma pequena porcentagem do número total de casos.

Os parâmetros microbiológicos observados neste trabalho foram divergentes ao encontrado por Rosa et al. (2008) e Almeida et al. (2017) ao avaliarem os aspectos higiênico-sanitários nas escolas públicas do município de ManacapuruAM e Bom Jesus, Colônia do Gurgueira-PI respectivamente, observaram que as práticas de higiene durante a produção de preparações à base de carne, apresentaram-se insatisfatórias evidenciando a necessidade de melhoria do processo de produção, visando as condições de saúde da população estudantil assistida.

\section{Conclusão}

Os filés de pescado analisados apresentaram boas características nutricionais, estando dentro dos parâmetros microbiológicos preconizados pela Agência Nacional de Vigilância Sanitária (ANVISA), portanto, aptos para serem consumidas pela comunidade estudantil do Estado do Amazonas.

\section{Referências bibliográficas}

Ackman, R. 1989. Nutritional composition of fats in seafoods. Progress in food \& nutrition science, 13(3-4), 161-289.

Ahmed, F. E. 1991. Seafood Safety: Committee on evaluation of the safety of fishery products. Food and Nutrition Board, Washington National Academy Press.

Almeida, S. R. R., Cardoso, J. d. F. S., Araujo, J. I. M., da Silva, A. L. A., de Araujo, J. M., da Rocha, R. B. \& dos Santos, G. R. 2017. Aspectos higiênico-sanitários da merenda escolar nas cidades de Bom Jesus e Colônia do Gurgueia-PI. PUBVET, 11, 1-102.

AOAC. 1995. Official methods of analysis of AOAC International. Arlington, Va.: AOAC Intl. pv (loose-leaf).

Brasil. 2001. Regulamento técnico sobre padrões microbiológicos para alimentos. Resolução$\mathrm{RDC} \mathrm{n}^{\circ} 12$ : de 02/01/01. Agência Nacional de Vigilância Sanitária-Anvisa, 2001. Diário Oficial, Brasília.

Brasil. 2003. Métodos Analíticos Oficiais para Análises Microbiológicas para Controle de Produtos de Origem Animal e Água: Instrução 
Normativa $\mathrm{n}^{\circ}$ 62, de 26/08/2003: Diário Oficial da União Brasília.

Carvalho, F. M. 1980. Composição química e reprodução do mapará (Hypophthalmus edentatus Spix, 1829) do lago do Castanho, Amazonas. (Siluriformes, Hypophthalmidae) (). Acta Amazonica, 10, 379-389.

Contreras-Guzmán, E. S. 1994. Bioquímica de pescados e derivados: FUNESP.

Costa, T. d., Oshiro, L. M. Y. \& Silva, E. (2010). $\mathrm{O}$ potencial do mapará Hypophthalmus spp.(Osteichthyes, Siluriformes) como uma espécie alternativa para a piscicultura na Amazônia. Bol Inst Pesca, 36(3), 165-174.

Dams, R. I., Beirão, L. H. \& Teixeira, E. 1996. Avaliação da qualidade microbiológica da pescadinha (Cysnoscion striatus) inteira e em filés nos principais pontos críticos de controle de uma indústria de pescado congelado. Boletim do Centro de Pesquisa de Processamento de Alimentos, 14(2).

Fao. 2004. El Estado mundial de la pesca y la acuicultura, 2004: Food \& Agriculture Org.

Farias, M. d. C. A. \& Freitas, J. d. A. 2008. Qualidade microbiológica de pescado beneficiado em indústrias paraenses. Revista do Instituto Adolfo Lutz (Impresso), 67(2), 113-117.

IAL, I. A. L. 2008. Métodos físico-químicos para análise de alimentos. São Paulo: ANVISA.

Landgraf, M. 1996. Deterioração microbiana de alimentos. Franco, BDG; Landgraf, $M$. Microbiologia dos alimentos. São Paulo: Editora Atheneu, 93-108.

Mukundan, M. K., Antony, P. D. \& Nair, M. R. 1986. A review on autolysis in fish. Fisheries Research, 4(3), 259-269.

NEPA, Núcleo de Estudos e Pesquisas e em Alimentação. 2011. Tabela de Composição de Alimentos-TACO: NEPA/UNICAMP.

Campinas:

Rall, V. L. M., Cardoso, K. \& Xavier, C. 2008. numeração de coliformes termotolerantes em pescados frescos e congelados. PUBVET, Londrina, 2(39).

Rosa, M. S., de Negreiros, S. R. F., Sebra, L. M. A. J. \& Stamford, T. L. M. 2008. Monitoramento de tempo e temperatura de distribuição de preparações à base de carne em escolas municipais de Natal (RN), Brasil Monitoring exposure time and distribution temperature of meat-based meals served in municipal schools in Natal, Brazil. Revista de Nutrição, 21(1), 21-28.

Santiago, L., Melo, J. K., Sila, F. G. \& Silva, E. V. C. 2016. Avaliação físico-químicas do mapará (Hypophthalmus marginatus) comercializado no município de Cametá-PA. In: $56^{\circ}$ Congresso Brasileiro de Química.

Silva, M. L. d., Matté, G. R. \& Matté, M. H. 2008. Aspectos sanitários da comercialização de pescado em feiras livres da cidade de São Paulo, SP/Brasil. Revista do Instituto Adolfo Lutz (Impresso), 67(3), 208-214.

Souza, F. d. C. d. A., Jesus, R. S. d., Duncan, W. L. P. \& Aguiar, J. P. L. 2013. Efeito do congelamento na composição química e perfil de aminoácidos da carne mecanicamente separada de peixes amazônicos. Revista PanAmazônica de Saúde, 4, 57-61.

Stansby, M. \& Olcott, H. 1968. Composición del pescado. Tecnología de la Industria Pesquera. Zaragoza: Acribia, Acribia. Zaragoza, p. 391402, 391-402.

Vasconcelos, E. L. Q., Viana, A. P. \& Oliveira, P. R. 2014. Avaliação microbiológica do pirarucu (Arapaima gigas) salgado seco comercializado em feiras da Cidade de Manaus e Fonte Boa, Amazonas. PUBVET, 8, 0340-0443.

Veit, J. C., Freitas, M., Reis, E., Moore, O., Finkler, J., Boscolo, W. \& Feiden, A. 2012. Development and characterization of chocolate and carrot cakes with nile tilapia (Oreochromis niloticus). Alimentos e Nutrição Araraquara, 23(3), 434.

Vieira, R. H. S. d. F., Rodrigues, D. d. P., Barreto, N. S. E., Sousa, O. V. d., Tôrres, R. C. d. O., Ribeiro, R. V., . . Madeira, Z. R. 2004. Microbiologia, higiene e qualidade do pescado: teoria e prática Microbiologia, higiene e qualidade do pescado: teoria e prática

Yeannes, M. a. I. \& Almandos, M. a. E. 2003. Estimation of fish proximate composition starting from water content. Journal of Food Composition and Analysis, 16(1), 81-92.

\section{Article History:}

Received 9 February 2018

Accepted 12 March 2018

Available online 30 April 2018

License information: This is an open-access article distributed under the terms of the Creative Commons Attribution License 4.0, which permits unrestricted use, distribution, and reproduction in any medium, provided the original work is properly cited. 